Case Report

\title{
Management of Avulsed Teeth using Fixed Orthodontics Appliances and Fiber Splint: A Case Series
}

\author{
Soegeng Wahluyo, Seno Pradopo, Tania Saskianti, Wahyudi Sudarsono, Putu Harlia Ernestine Harta \\ Department of Pediatric Dentistry, \\ Faculty of Dental Medicine, Universitas Airlangga, \\ Surabaya - Indonesia
}

\begin{abstract}
Background: Tooth avulsion is defined as the complete displacement of the tooth out of its alveolar socket. The treatment of choice is immediate replantation or if that is not possible, placement of the tooth in an appropriate storage media. Purpose: This paper reported of two cases of treatment management in children who experienced avulsion of the upper central incisor. The first and the second case, discuss about tooth that has been avulsed for 20 hours of unsterilized storage and 4 hours of storage in milk. Case Management: Replantation of those teeth were done by root canal treatment outside the oral cavity first. For the first case followed by splinting method using fixed orthodontics appliances, and for the second case followed by splinting using fiber and composite. Discussion: Evaluation were done every week, until 3 months after replantation to see the the success of the treatment. The progress of two cases with different situation showed good results. Conclusion: The case showed that the tooth that has been avulsed for 20 hours of unsterilized storage and 4 hours of storage in milk could have good prognosis.
\end{abstract}

Keywords : avulsed tooth, replantation, splinting

Correspondence: Soegeng Wahluyo, Department of Pediatric Dentistry, Faculty of Dental Medicine, Universitas Airlangga, Jl. Prof. Dr. Moestopo 47 Surabaya 60132 - Indonesia, Phone: 031-5030255 Email: soegeng-w@fkg.unair.ac.id

\section{INTRODUCTION}

Tooth avulsion is total displacement of the tooth out of its alveolar socket. The incidence even higher in children between 8 and 12 years old due to bicycle, skateboard, and sports accidents. Management of avulsed permanent dentition often presents a challenge. Clinical studies have shown that the prognosis is best for teeth replanted within 5 minutes after avulsion, yet such optimal treatments not always possible. ${ }^{1,2}$

This case report discussed about two case management of avulsed upper central incisor in children, pre-treated with different solution and splinted using orthodontic appliance and using fiber splint. This paper reported of two cases of treatment management in children who experienced avulsion of the upper central incisor. The first and the second case, discuss about tooth that has been avulsed for 20 hours of unsterilized storage and 4 hours of storage in milk.

\section{CASES}

Case 1

An 11-year-old boy came to the Pediatric Dentistry Specialist Clinic of Airlangga University Dental Hospital due to avulsed tooth. The avulsed teeth were accidentally washed, and was taken to the clinic 20 hours later. On examination, the patient showed no neurological symptoms and extra oral injury. The tooth had crown and root fracture and closed apex. There were blood clots in the socket of 21 and injured upper lip. The patient had mixed dentition, overjet $6 \mathrm{~mm}$, class I molar relations and good oral hygiene. From panoramic findings, there were no hard tissue abnormalities around the avulsed tooth. 

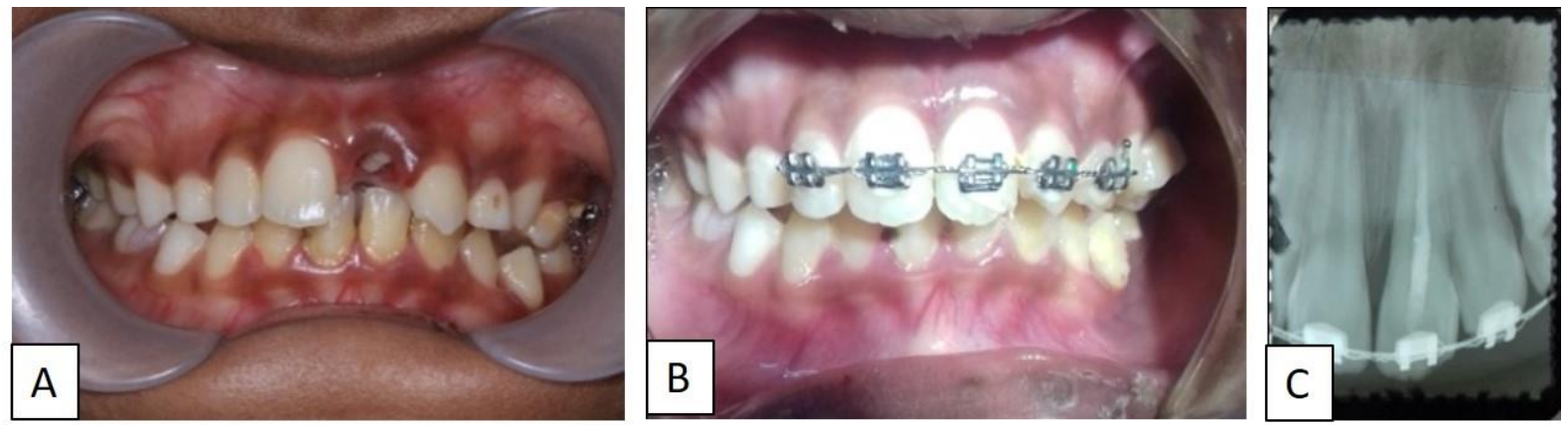

Figure 1. (a) Intra Oral Examination before treatment in the first case; (b) Intra Oral Examination after treatment using orthodontic appliance; (c) 3-month evaluation periapical radiographs.

The procedure was first soaked the tooth in saline solution then root canal treatment was performed outside the oral cavity. Subsequently, infiltration anesthesia (scandonest 2\%) was performed, blood clots were taken from inside the socket. The tooth was splinted using fixed orthodontics appliances in 12, 11, 21, 22, and 63 and SS wire 0.16 , then ligated with wire ligature for tooth fixation. Amoxicillin $250 \mathrm{mg}$ and Mefenamic Acid $250 \mathrm{mg}$ was prescribed orally to prevent infection.

Dental evaluation was carried out at 1 week, 1 month and 3 months after replantation. On the 3-month evaluation, there were no pain, no tooth mobility, good incisal alignment, no gingival swelling and no radiolucent at the apical teeth so that splinting was removed.

\section{Case 2}

An 9-year-old girl was referred to the Pediatric Dentistry Specialist Clinic of Universitas Airlangga Dental Hospital due to avulsed tooth. The trauma occurred 4 hours ago while the child was playing. Her parents kept the avulsed tooth in a bottle of milk and brought it to the clinic. The patient had no medical history and no neurological damage. The intraoral examination showed that the tooth 21 was avulsed and there was laceration of the palatal mucosa. Panoramic radiographs revealed no alveolar bone fracture or other hard tissue injuries, the crown had enamel fracture and open apex. The patient had mixed dentition, overjet $5 \mathrm{~mm}$, and severe carious lesions due to poor oral hygiene.

The socket of the tooth was first gently rinsed with saline solution under infiltration anesthesia (scandonest 2\%). The root of the tooth was cleaned carefully to remove necrotic and dried remnants of periodontal tissue. Extra-oral endodontic treatment was performed, the root canals were filled with $\mathrm{CaOH}$, and the tooth was replanted slowly with slight pressure. Glass ionomer cement restoration were used to restore the access cavity. The tooth was stabilized using a fiber splint and the acid-etch composite resin technique.

The patient instructed to maintain good oral hygiene, soft diet and use a chlorhexidine mouth rinse. Amoxicillin $500 \mathrm{mg}$ was prescribed for five days, three times/day. The patient was followed up in 1 day and the splint in a good condition. Three months after replantation, there were no pain, no tooth mobility, good incisal alignment, no gingival swelling, no radiolucent at the apical teeth, and the periodontal ligament began to form. The patient also had been able to eat normally. 

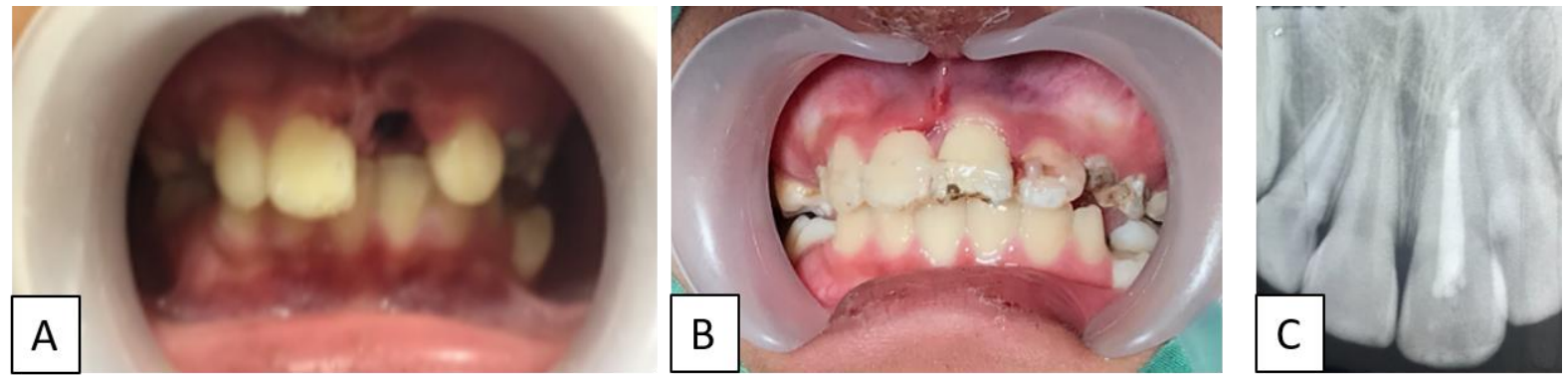

Figure 1. (a) Intra Oral Examination before treatment in the second case; (b) Intra Oral Examination after treatment using orthodontic appliance; (c) 8-month evaluation periapical radiographs.

\section{DISCUSSION}

In clinical studies, teeth replanted within 5 minutes after avulsion had the best prognosis. The avulsed tooth should be replanted immediately or should be stored in a physiological medium such as saline for only a short period before replantation. The replanted tooth should be splinted flexibly to the adjacent teeth for 7 to 10 days to enhance periodontal healing. If the tooth apex is closed or almost closed, prophylactic root canal treatment should be carried out on the day of splint removal to prevent the onset of inflammatory root resorption. ${ }^{1-3}$

In the first case, the avulsed incisor had a nearly closed apex, had been air-dried for a prolonged period and the root canal treatment of the avulsed incisor was also filled extra orally. Tufenkci, et al (2017) showed that further drying and handling of the root surface was unlikely to worsen the prognosis. ${ }^{4}$

The storage and transport media during the extra-oral time are also being determinant of successful treatment. The tooth should be maintained in a suitable media, such as HBSS, saline, milk, or saliva until it is replanted by a dentist. Milk is a good choice due to its osmolarity and $\mathrm{pH}$ and has been shown to preserve periodontal ligament cells for up to 8 hour. ${ }^{1,4}$

The splinting type can be temporary splinting or permanent splinting. Orthodontic retention can also be considered as a type of permanent splinting. In the first case, we used orthodontic appliance for treat avulsed tooth. Permanent splinting is used during the rehabilitation of the complex oral cavity where the tooth is experiencing high mobility, especially when the supporting tooth has minimal periodontal support. If the teeth are not splinted, there will be a danger of continued tooth mobility. Some studies show splinting can increase tissue resistance to further periodontal damage and accelerate the healing response. ${ }^{4-6}$

In the second case, we used fiber splint with etch-acid composite resin for treat avulsed teeth. This splint can function for several months or even years. This application technique is similar to that of composite resin restorations using etching-acid technique. ${ }^{4,5}$

Ankyloses (replacement resorption) is defined as a fusion of the alveolar bone and root surface. During ankyloses, the tooth root merges with the remodeled normal bone and is gradually replaced with bone. The latter can be recognized within 4-8 weeks after replantation. In the present case, although the first and second case was stored in saline and milk for the whole extra-alveolar duration, some parts of the periodontal ligament tissues seemed to have become necrotic because of the prolonged extra-alveolar period. ${ }^{4,7}$

During 3 months of follow-up ankyloses was not evident clinically or radiographically. It is important to highlight that avulsion is a severe dental injury, if it is managed immediately and appropriately, the avulsed teeth can be preserved and be functional for some years. The case showed that the tooth that has been avulsed for 20 hours of unsterilized storage and 4 hours of storage in milk could have good prognosis.

\section{REFERENCES}

1. Andersson L, Jens O, Day P, et al. International Association of Dental Traumatology guidelines for the management of traumatic dental injuries: 2 . Avulsion of permanent teeth. 2012:88-96. doi:10.1111/j.16009657.2012.01125.x 


\section{Indonesian Journal of Dental Medicine}

Volume 1 Issue 1 2018; 14-17

2. Emerich, Czerwinska O. Immediate selfreplantation of an avulsed permanent mandibular incisor - a case report. Dent Traumatol. 2010; 26(5): 443-446. doi:10.1111/j.1600-9657.2010.00921.x

3. Trope M. Clinical Management of Avulsed Tooth : Present Strategies and Future Directions. Dent Traumatol. 2002;18(1):1-11.

4. Tufenkci P, Canbolat F, Celikten B, Sevimay S. Reimplantation of an avulsed tooth after five hours of storage in milk: One-year follow-up case report. 2017;4(4):48-51. doi:10.5430/crim.v4n4p48
5. Requião S, Rocha T, Moro A, Moresca RC, Sydney G. Orthodontic treatment in patients with reimplanted teeth after traumatic avulsion : A case report. 2010;15(4):1-10.

6. Singh M, Singh N, Dhiman RK, Kumar D. External replacement resorption in an avulsed reimplanted permanent incisors. 2013;5(1):2-6. doi:10.4103/2231-0754.134134

7. Moradian, Badakhsh R. Replantation of an Avulsed Maxillary Incisor after 12 Hours: Three-Year Follow-Up. Iran Endod J. 2013;8(1):33-36. 